\title{
Identification of genes and pathways associated with multiple organ dysfunction syndrome by microarray analysis
}

\author{
CHANGWEI GU, WANHAI QIAO, LINA WANG, MINMIN LI and KANG SONG
}

Emergency Department, The Second Affiliated Hospital of Xi'an Jiaotong University, Xi'an, Shaanxi 710004, P.R. China

Received October 19, 2016; Accepted November 21, 2017

DOI: $10.3892 / \mathrm{mmr} .2018 .8973$

\begin{abstract}
Multiple organ dysfunction syndrome (MODS) is characterized by the development of progressive physiological dysfunction of $\geq 2$ organs or organ systems and is responsible for the majority of the morbidity and mortality among patients in intensive care units. The aim of the present study was to investigate the potential genes and pathways associated with MODS. The microarray dataset GSE60088 was downloaded from the Gene Expression Omnibus and used to identify differentially expressed genes (DEGs) between organ tissues (lung, liver and kidney) obtained from a murine model of MODS and healthy controls. The interactions between DEGs in lungs, liver and kidneys were revealed by Kyoto Encyclopedia of Genes and Genomes pathway enrichment analysis. Furthermore, protein-protein interaction (PPI) data for DEGs were obtained from the Search Tool for the Retrieval of Interacting Genes and a PPI network was constructed. Additionally, DEGs that were common among the three organs were screened and transcription factors that regulated them were predicted using the iRegulon plugin. A total of 943, 267 and 227 DEGs were identified in lung, liver and kidney samples, respectively, between mice with MODS and healthy controls. In lung and liver samples, two pathways that were enriched with DEGs were identified and were common between lung and liver samples, including 'cytokine-cytokine receptor interaction' and 'Jnk-STAT signaling pathway', and examples of DEGs associated with these pathways include $\mathrm{C}-\mathrm{X}-\mathrm{C}$ motif chemokine ligand (Cxcl)1 and $\mathrm{Cxcl10}$, and signal transducer and activator of transcription (Stat)1, respectively. Furthermore, two common pathways were identified in liver and kidney samples, which included 'MAPK signaling pathway' and 'p53 signaling pathway', and DEGs associated with these pathways included growth arrest and DNA damage-inducible $\alpha$. A total of 18
\end{abstract}

Correspondence to: Dr Wanhai Qiao, Emergency Department, The Second Affiliated Hospital of Xi'an Jiaotong University, 157 Xiwu Road, Xi'an, Shaanxi 710004, P.R. China

E-mail: wanhqiao@yeah.net

Key words: multiple organ dysfunction syndrome, differentially expressed genes, pathway, network, transcription factor
DEGs were common among lung, liver and kidney tissues, including CCAAT/enhancer binding protein $\beta(\mathrm{Cebpb})$ and olfactomedin-like 1 (Olfml1). Cebpb modulated various other DEGs, such as Cxcl1, and Olfml1 was regulated by Stat5A. These genes and pathways may serve roles in the progression of MODS and may be considered to be potential therapy targets for MODS.

\section{Introduction}

Multiple organ dysfunction syndrome (MODS) is characterized by the development of progressive physiological dysfunction of at least two organs or organ systems that is induced by various acute physiological damages, including trauma, burns, shock and severe infection $(1,2)$. MODS is responsible for the majority of the morbidity and mortality among patients in intensive care units $(3,4)$. MODS primarily affects the lungs, liver, kidney and heart (3).

Multiple factors contribute to the pathological process of MODS; however, the immunoinflammatory system is considered to serve the most important role in the pathogenesis of MODS $(5,6)$. In the early stage of MODS, abundant release of proinflammatory cytokines, including tumor necrosis factor- $\alpha$ and interleukin (IL)-1 $\beta$, upregulates the expression of vascular cell adhesion molecule 1 and endothelial leukocyte adhesion molecule 1 , and enhances the adhesion of monocytes and T cells to endothelial cells $(7,8)$. During later stages of MODS, damage to the endothelium activates innate inflammatory cells in the interstitial region of affected organs and parenchymal injury occurs (5). Furthermore, other factors have been reported to be closely associated with the pathogenesis of MODS. For example, mitochondrial dysfunction is implicated in organ injury through accelerated secretion of oxidants and promotion of cell death (9). Increased heme oxygenase- 1 and reduced non-specific $\delta$-aminolevulinate synthase has been reported in septic animal models of MODS (10). A recent study demonstrated that tissue fibrosis is a common pathogenic pathway that is implicated in organ injury or failure, and multiple molecules are involved in this pathway, including mitogen-activated protein kinase (MAPK), rho-associated protein kinase and transforming growth factor (TGF)- $\beta$ (11).

Gene microarrays have been widely used to investigate the nosogenesis of various diseases, including psychiatric disorders, eczema, sepsis and multiple cancer types (12-16). 
A recent study by Gharib et al (17) focused on processes that were frequently activated in at-risk organs and identified various putative factors implicated in early MODS based on a microarray analysis. However, numerous genes associated with the progression of MODS have not yet been identified and underlying gene regulatory mechanisms remain to be elucidated. In the present study, a gene expression profile (GSE60088) submitted by Gharib et al (17). was used to identify differentially expressed genes (DEGs) in lung, liver and kidney tissues of mouse models of MODS. The functions of, and interactions between, DEGs were subsequently analyzed. Furthermore, DEGs that were common among lung, liver and kidney tissues were determined, and the transcription factors (TFs) regulating them were predicted. The results of the present study may contribute to the understanding of the molecular mechanisms underlying MODS.

\section{Materials and methods}

Affymetrix microarray data. The raw gene expression profile dataset GSE60088 was downloaded from the public Gene Expression Omnibus database (http://www.ncbi.nlm .nih.gov/geo/) (17). Microarray data were generated by the Affymetrix Mouse Genome 430 2.0 Array platform (accession no. GPL1261; Affymetrix; Thermo Fisher Scientific, Inc., Waltham, MA, USA). A total of 27 tissue samples from a murine model of sepsis-induced MODS or controls were included in the dataset, including 5 lung samples from mice with MODS, 4 healthy lung samples, 5 liver samples from mice with MODS, 3 healthy liver samples, 5 kidney samples from mice with MODS and 5 healthy kidney samples. The murine model of sepsis-induced MODS was established by exposure to a combination of mechanical ventilation and Staphylococcus aureus pneumonia (17).

CEL files and probe annotation files were downloaded from the Affymetrix database. Gene expression data from all samples were preprocessed with background correction, quantile normalization and calculation of gene expression using the robust microarray analysis algorithm of the Affy package (v1.54.0) (18) downloaded from Bioconductor (http://www.bioconductor.org/). Subsequently, annotations file org.Mm.eg.db and mouse4302.db in R language (v3.1.3) were downloaded from Bioconductor, and were used for the conversion of probe IDs to gene symbols $(19,20)$. If one gene symbol was matched by multiple probe IDs, then the mean expression value was selected as the expression level of this gene.

Identification of DEGs. The linear models for microarray data (Limma; v3.32.2) package (21) in R language (v3.1.3) was used to identify DEGs between samples from mice with MODS and healthy lung, liver and kidney control samples. The P-value for the differential expression of each gene was calculated using an unpaired t-test and adjusted to the false discovery rate (FDR) using the Benjamini-Hochberg method (22). Only genes with $F D R<0.05$ and $\log _{2}$ fold changel $\geq 1$ were selected as DEGs. Additionally, DEGs identified in each type of tissue were clustered using gplots package (v3.5.0) in the R software (v3.1.3) (23). Ultimately, heatmaps were generated with the pheatmap (24) package in R language (v3.1.3).
Pathway enrichment analysis. Kyoto Encyclopedia of Genes and Genomes (KEGG) pathway enrichment analysis of DEGs in lung, liver and kidney samples was performed using the Database for Annotation, Visualization and Integrated Discovery (DAVID; http://david.abcc.ncifcrf.gov/) (25). The $\mathrm{P}$-value was calculated by Fisher's exact test, and $\mathrm{P}<0.05$ and gene count $>2$ were set as the cut-off criteria.

Construction of protein-protein interaction (PPI) networks. The PPI networks of DEGs in lung, liver and kidney tissues were identified using the Search Tool for the Retrieval of Interacting Genes (STRING; http://string-db.org/) database, which integrates various known and predicted protein associations (26). A combined score $>0.7$ was set as the cut-off criterion. Subsequently, the PPI network was visualized by Cytoscape software (v3.3.0; http://cytoscape.org/) (27). In the network, a node represents a protein product of a gene and lines represent interactions between proteins. The degree of each node represents the number of nodes that interact with a given node. The greater the degree, the closer the connection to other nodes.

Analysis of the DEGs identified in lung, liver and kidney tissues. Upregulated and downregulated DEGs that were common among lung, liver and kidney tissues were identified using the online tool Venny (v2.0; http://bioinfogp.cnb. csic.es/tools/venny/index.html) and Venn diagrams were constructed based on the data.

Subsequently, TFs regulating DEGs in all samples were analyzed using the plugin iRegulon (v1.3) in Cytoscape (28). Minimum identity between orthologous genes was set at 0.05 and maximum FDR on motif similarity was set as 0.001 . The larger the normalized enrichment score (NES) for the output, the more reliable the regulatory association. In the present study, regulatory associations with NES $>5$ were selected to construct the regulatory network visualized by Cytoscape.

\section{Results}

Identification of DEGs. Following data normalization, median gene expression in each sample was similar (Fig. 1), confirming that the normalized data was suitable for further analyses. Based on the cut-off criteria, a total of 943 DEGs (618 upregulated and 325 downregulated) were identified in lung samples from mice with MODS, 267 DEGs (185 upregulated and 82 downregulated) were identified in liver samples from mice with MODS and 227 DEGs (161 upregulated and 66 downregulated) were identified in kidney samples from mice with MODS. Clustering heatmaps demonstrated that samples from mice with MODS were different compared with the healthy controls (Fig. 2).

KEGG pathway enrichment analysis of DEGs. To investigate the biological functions of the identified DEGs, KEGG pathway enrichment analysis was conducted for DEGs in lung, liver and kidney samples. According to the pathway enrichment analysis, in lung samples from mice with MODS, upregulated DEGs were primarily involved in the following pathways implicated in immunity: 'Cytokine-cytokine receptor interaction', including $\mathrm{C}-\mathrm{X}-\mathrm{C}$ motif chemokine 
A

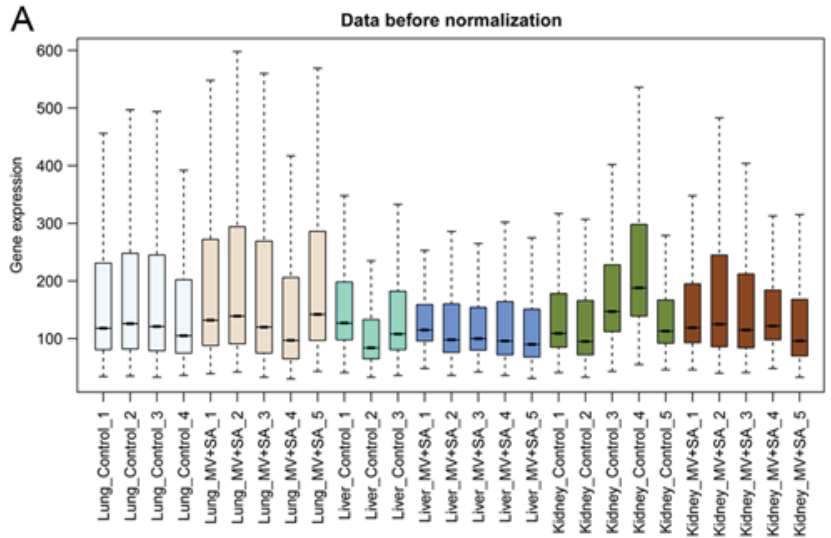

B

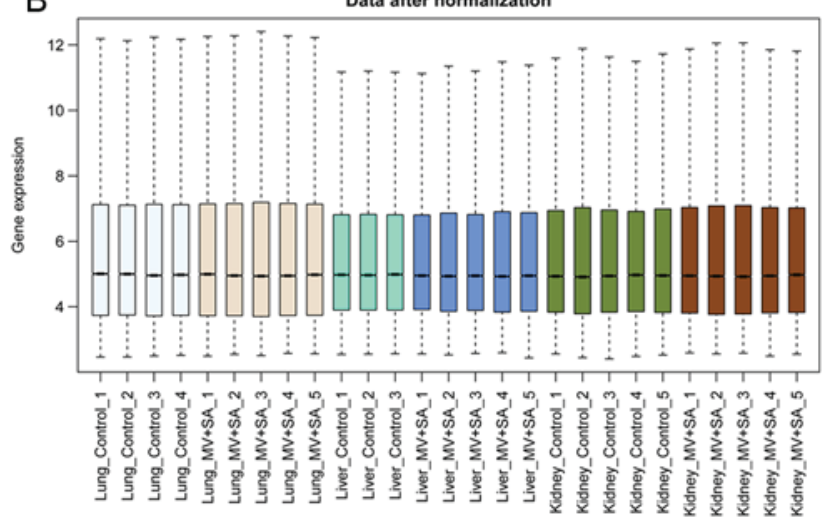

Figure 1. Boxplots of gene expression profiles of lung, liver and kidney samples. (A) Gene expression profile of each sample prior to the data normalization. (B) Gene expression of each sample following data normalization. The ordinate represents total gene expression value and the abscissa represents tissue samples of lungs, liver and kidneys from a murine model of MODS and healthy mice. The black line in the rectangular block represents the median gene expression. MODS, multiple organ dysfunction syndrome; MV+SA, murine model of MODS induced by exposure to a combination of mechanical ventilation and Staphylococcus aureus pneumonia.

A

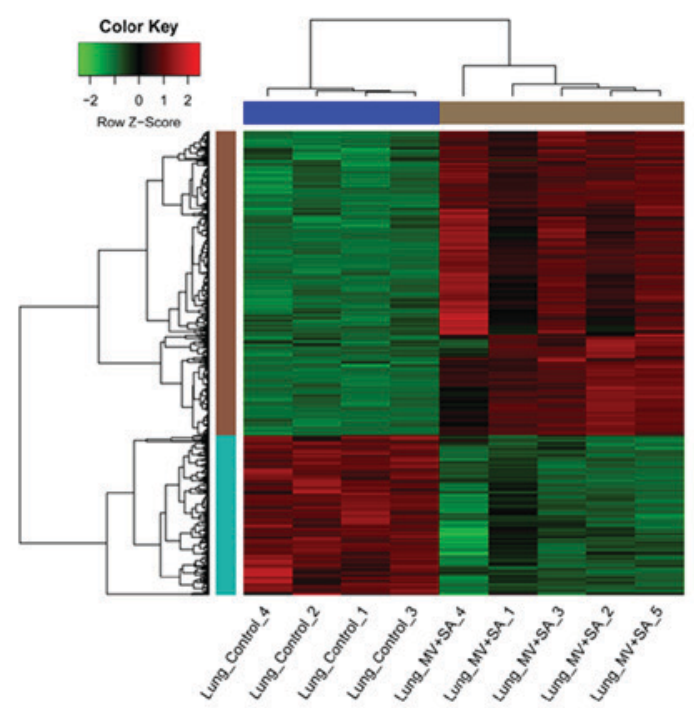

B

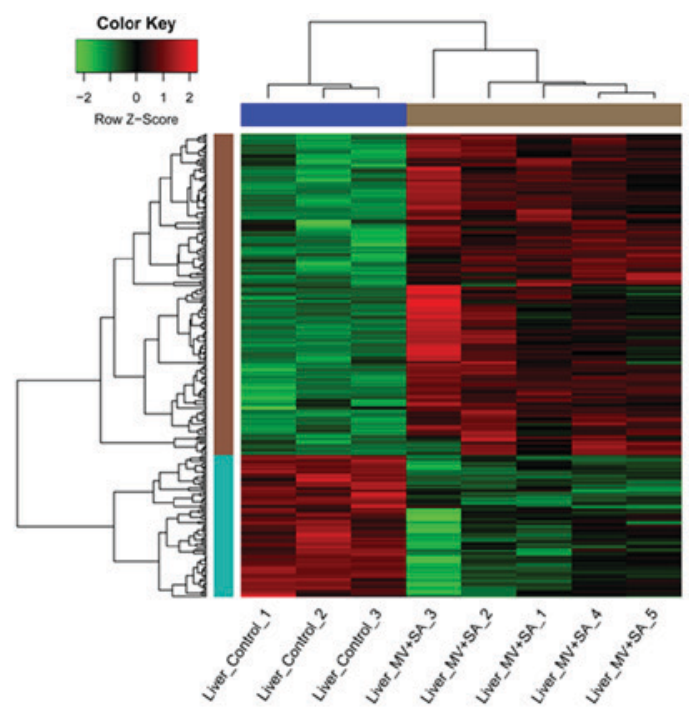

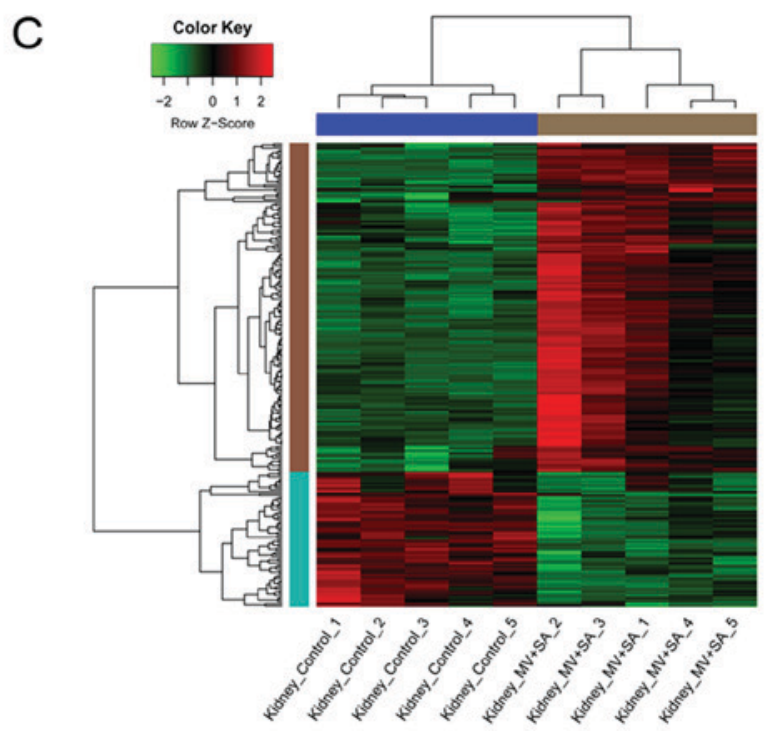

Figure 2. Heat maps of DEGs in tissue samples from mice with MODS and healthy controls. DEGs in (A) lung (B) liver and (C) kidney samples from mice with MODS compared with the healthy controls. Each row represents a gene and each column represents a sample. Red color represents upregulation and green represents downregulation of genes. DEGs, differentially expressed genes; MODS, multiple organ dysfunction syndrome; MV+SA, murine model of MODS induced by exposure to a combination of mechanical ventilation and Staphylococcus aureus pneumonia. 
Table I. Pathways enriched with DEGs in lung samples from mice with MODS compared with healthy controls.

A, Pathways that upregulated DEGs in lung samples from MODS mice were enriched in

\begin{tabular}{|c|c|c|c|}
\hline Pathway & P-value & Gene count & DEGs \\
\hline $\begin{array}{l}\text { mmu04060: Cytokine-cytokine receptor } \\
\text { interaction }\end{array}$ & $7.75 \times 10^{-22}$ & 52 & $\begin{array}{l}\text { Il17Ra, Cxcl10, Il18Rap, Cd40, Osm, Inhba, Tnfrsf9, } \\
\text { Ccr7, Cxcl1, IL-6... }\end{array}$ \\
\hline mmu04062: Chemokine signaling pathway & $2.32 \times 10^{-16}$ & 39 & $\begin{array}{l}\text { Cxcl1, Cxcl10, Stat1, Ccr5, Cxcl13, Cxcl16, Ccr2, } \\
\text { Jak2, Jak3, Xcl1... }\end{array}$ \\
\hline $\begin{array}{l}\text { mmu04620: Toll-like receptor } \\
\text { signaling pathway }\end{array}$ & $4.06 \times 10^{-12}$ & 25 & $\begin{array}{l}\text { Ccl3, Tnf, Cxcl10, Myd88, IL-6, Cd40, Stat1, Ripk1, } \\
\text { Irf7, Cd14... }\end{array}$ \\
\hline $\begin{array}{l}\text { mmu04621: NOD-like receptor } \\
\text { signaling pathway }\end{array}$ & $8.60 \times 10^{-11}$ & 19 & $\begin{array}{l}\text { Cxcl1, IL-6, Tnf, Ccl2, Cxcl2, Ccl8, Nfkbia, Nlrp3, } \\
\text { Ccl5, Ccl7... }\end{array}$ \\
\hline mmu04630: Jak-STAT signaling pathway & $7.80 \times 10^{-7}$ & 23 & $\begin{array}{l}\text { Csf3, Socs1, Pim1, Stat1, Il7R, Stat2, Csf2Rb, Jak2, } \\
\text { Pik3R5, Jak3... }\end{array}$ \\
\hline
\end{tabular}

B, Pathways that downregulated DEGs in lung samples from MODS mice were enriched in

\begin{tabular}{|c|c|c|c|}
\hline Pathway & P-value & Gene count & DEGs \\
\hline mmu00982: Drug metabolism & $5.02 \times 10^{-8}$ & 12 & $\begin{array}{l}\text { Gstm1, Gsta2, Gsta3, Cyp2D22, Adh1, Fmo1, Fmo2, } \\
\text { Fmo3, Maob, Aldh3A1... }\end{array}$ \\
\hline $\begin{array}{l}\text { mmu00980: Metabolism of xenobiotics } \\
\text { by cytochrome P450 }\end{array}$ & $1.68 \times 10^{-6}$ & 10 & $\begin{array}{l}\text { Gstm1, Gsta2, Gsta3, Cyp2F2, Cyp1A1, Adh1, Gstt1, } \\
\text { Ephx1, Cyp2E1, Aldh3A1 }\end{array}$ \\
\hline mmu04512: ECM-receptor interaction & $5.56 \times 10^{-4}$ & 8 & Lama2, Col4A4, Tnxb, Npnt, Itga8, Vtn, Thbs3, Chad \\
\hline mmu05414: Dilated cardiomyopathy & $1.03 \times 10^{-3}$ & 8 & Lama2, Actc1, Adrb1, Adcy8, Pln, Itga8, Myh7, Tnni3 \\
\hline mmu00340: Histidine metabolism & $8.94 \times 10^{-3}$ & 4 & Hnmt, Acy3, Maob, Aldh3A1 \\
\hline
\end{tabular}

DEGs, differentially expressed genes; MODS, multiple organ dysfunction syndrome. DEGs were listed in the table based on P-value and llog2 $\mathrm{FCl}$ value of these DEGs.

ligand (Cxcl)1, Cxcl10 and IL-6 DEGs; 'toll-like receptor signaling pathway', including Cxcl10, IL-6 and signal transducer and activator of transcription (Stat)1 DEGs; and 'Jak-STAT signaling pathway', including colony-stimulating factor 3, IL-6 and Stat1 DEGs. The downregulated DEGs in lung samples from mice with MODS were involved in the following pathways: 'Metabolism of xenobiotics by cytochrome P450', including glutathione S-transferase $\alpha 2$, aldehyde dehydrogenase 3 family member A1 (Aldh3A1) and cytochrome P450 family 1 subfamily A member 1 DEGs; 'ECM-receptor interaction', including laminin subunit $\alpha 2$ and collagen type IV $\alpha 4$ chain DEGs; and 'histidine metabolism', including histamine N-methyltransferase and Aldh3A1 DEGs (Table I).

In liver samples from mice with MODS, the upregulated DEGs were primarily enriched in the following pathways: 'Jak-STAT signaling pathway', including Stat1 and Stat3 DEGs; 'cytokine-cytokine receptor interaction', including IL-17 receptor A and Cxcl10 DEGs; 'p53 signaling pathway', including serpin family $\mathrm{E}$ member 1 and growth arrest and DNA damage-inducible (Gadd) $\beta$ (Gadd45B) DEGs; and 'MAPK signaling pathway', including Jun proto-oncogene, AP-1 transcription factor subunit (Jun) and Gadd45B DEGs. The downregulated DEGs were primarily involved in the following pathways: 'Steroid hormone biosynthesis', including hydroxy- $\Delta$-5-steroid dehydrogenase, $3 \beta$ - and steroid $\Delta$-isomerase $2(\mathrm{Hsd} 3 \mathrm{~B} 2)$ and steroid $5 \alpha$-reductase 1 (Srd5A1) DEGs; 'nitrogen metabolism', including carbonic anhydrase (Car)5A and Car1 DEGs; and 'androgen and estrogen metabolism', including Hsd3B2 and Srd5A1 DEGs (Table II).

In kidney samples from mice with MODS, upregulated DEGs were primarily involved in the following pathways: 'MAPK signaling pathway', including dual-specificity phosphatase 1 and Jun DEGs; 'TGF- $\beta$ signaling pathway', including inhibin $\beta$ B subunit and SMAD family member 1 DEGs; and 'p53 signaling pathway', including thrombospondin 1 and Gadd $\alpha$ (Gadd45A) DEGs. The downregulated DEGs were primarily implicated in 'PPAR signaling pathway', including uncoupling protein 1, adiponectin, C1Q and collagen domain-containing, and fatty acid-binding protein 5 DEGs, and 'terpenoid backbone biosynthesis', including mevalonate diphosphate decarboxylase and isopentenyl-diphosphate $\Delta$ isomerase 1 DEGs (Table III).

Analysis of PPI networks. To investigate the interactions between DEGs, PPI networks for the DEGs in lung, liver and kidney samples were constructed. For DEGs in lung samples from mice with MODS, the PPI network consisted of 524 nodes and 2,703 interactions (Fig. 3A). In particular, Stat1 
Table II. Pathways enriched with DEGs in liver samples from mice with MODS compared with healthy controls.

A, Pathways that upregulated DEGs in liver samples from MODS mice were enriched in

\begin{tabular}{lccl}
\hline Pathway & P-value & Gene count & \multicolumn{1}{c}{ DEGs } \\
\hline mmu04630: Jak-STAT signaling pathway & $1.36 \times 10^{-2}$ & 6 & $\begin{array}{l}\text { Irf9, Osmr, Socs3, Il13Ra1, Stat1, Stat3 } \\
\text { Inhbb, Cxcl1, Tnfrsf1A, Osmr, Il13Ra1, Il17Ra, } \\
\text { mmu04060: Cytokine-cytokine receptor }\end{array}$ \\
$\begin{array}{llll}\text { interaction } \\
\text { mmu04115: p53 signaling pathway }\end{array}$ & $2.60 \times 10^{-2}$ & 7 & Cxcl10 \\
mmu04010: MAPK signaling pathway & $3.70 \times 10^{-2}$ & 7 & Rrm2, Serpine1, Gadd45B, Gadd45A \\
& & & Tnfrsf1A, Jun, Pla2G12A, Fgf21, Gadd45B, \\
& & &
\end{tabular}

B, Pathways that downregulated DEGs in liver samples from MODS mice were enriched in

\begin{tabular}{lccl}
\hline Pathway & P-value & Gene count & \multicolumn{1}{c}{ DEGs } \\
\hline mmu00140: Steroid hormone biosynthesis & $5.43 \times 10^{-4}$ & 4 & Hsd3B2, Cyp7A1, Hsd3B5, Srd5A1 \\
mmu00910: Nitrogen metabolism & $3.08 \times 10^{-3}$ & 3 & Car5A, Car1, Car3 \\
mmu00150: Androgen and estrogen metabolism & $6.29 \times 10^{-3}$ & 3 & Hsd3B2, Hsd3B5, Srd5A1 \\
\hline
\end{tabular}

DEGs, differentially expressed genes; MODS, multiple organ dysfunction syndrome.

Table III. Pathways enriched with DEGs in kidney samples from mice with MODS compared with healthy controls.

A, Pathways that upregulated DEGs in kidney samples from MODS mice were enriched in

\begin{tabular}{|c|c|c|c|}
\hline Pathway & P-value & Gene count & DEGs \\
\hline mmu04010: MAPK signaling pathway & $8.18 \times 10^{-4}$ & 10 & $\begin{array}{l}\text { Dusp5, Fos, Dusp4, Dusp1, Jun, Nr4A1, Fgf21, } \\
\text { Gadd45A, Myc, Ddit3 }\end{array}$ \\
\hline mmu04350: TGF- $\beta$ signaling pathway & $9.34 \times 10^{-3}$ & 5 & Inhbb, Smad1, Thbs1, Myc, Bmp6 \\
\hline mmu04115: p53 signaling pathway & $2.80 \times 10^{-2}$ & 4 & Cdkn1A, Pmaip1, Thbs1, Gadd45A \\
\hline mmu05200: Pathways in cancer & $3.33 \times 10^{-2}$ & 8 & Fos, Cdkn1 A, IL-6, Ptgs2, Jun, Fgf21, Myc, Stat3 \\
\hline $\begin{array}{l}\text { mmu04610: Complement and } \\
\text { coagulation cascades }\end{array}$ & $3.46 \times 10^{-2}$ & 4 & Fgg, Thbd, Fga, F3 \\
\hline
\end{tabular}

B, Pathways that downregulated DEGs in kidney samples from MODS mice were enriched in

\begin{tabular}{lccl}
\hline Pathway & P-value & Gene count & DEGs \\
\hline mmu03320: PPAR signaling pathway & $2.75 \times 10^{-2}$ & 3 & Ucp1, Adipoq, Fabp5 \\
mmu00900: Terpenoid backbone & $4.54 \times 10^{-2}$ & 2 & Mvd, Idi1 \\
biosynthesis & & & \\
\hline
\end{tabular}

DEGs, differentially expressed genes; MODS, multiple organ dysfunction syndrome.

(degree=99), IL-6 (degree=4) and Cxcl10 (degree=54) with higher degrees were identified (Table IV).

A total of 99 nodes and 179 interactions were included in the PPI network of DEGs from liver samples of mice with MODS (Fig. 3B). Notably, Jun (degree=16), Stat3 (degree=16), Stat1 (degree $=16)$ and Cxcl10 (degree=11) with higher degrees were identified (Table IV).

Analysis of DEGs in kidney samples from mice with MODS revealed that the PPI network contained 100 nodes and
229 interactions (Fig. 3C). Especially, Jun (degree=28), IL-6 (degree=23) and Stat3 (degree=17) with higher degrees were identified (Table IV).

DEGs that are common among lung, liver and kidney samples. The present study identified 17 upregulated DEGs that were common among lung, liver and kidney samples: ChaC glutathione-specific $\gamma$-glutamylcyclotransferase 1 ; predicted gene Gm20186; growth differentiation factor 15; lipocalin 2 (Lcn2); 
A

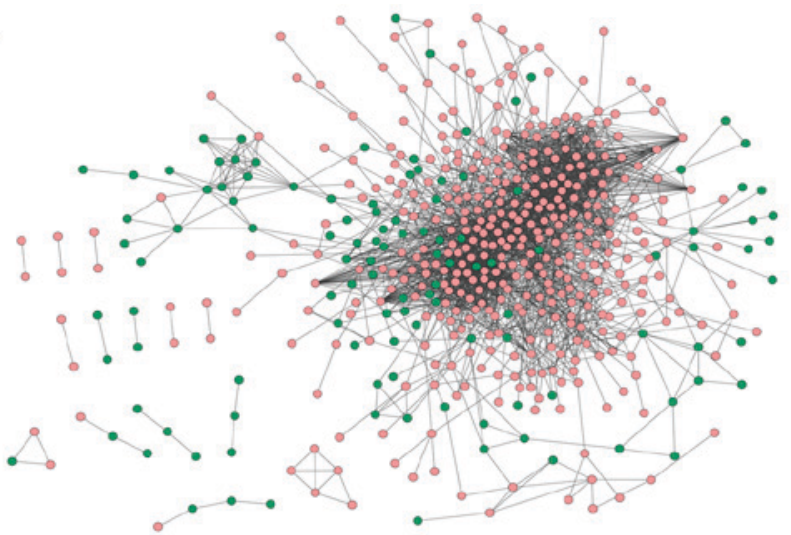

B
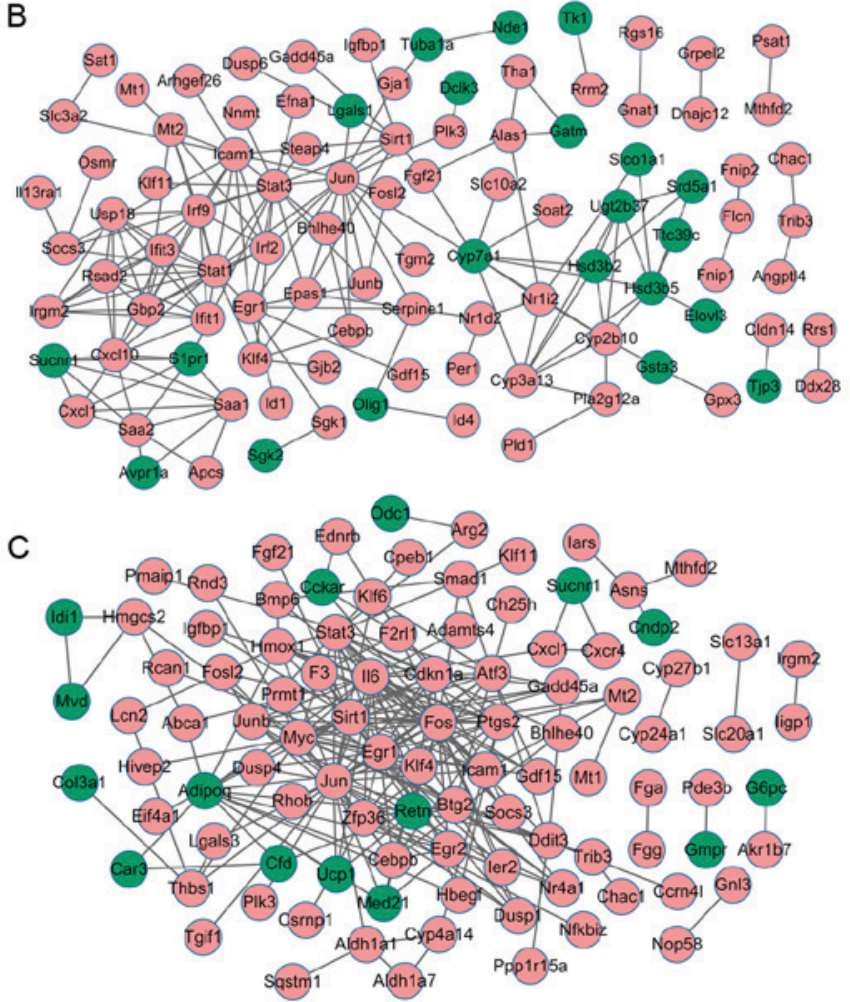

Figure 3. Protein-protein interaction network of DEGs compared with the healthy controls. The network of DEGs in (A) lung (B) liver and (C) kidney samples from mice with multiple organ dysfunction syndrome. Pink nodes represent upregulated genes and green nodes represent the downregulated genes. DEGs, differentially expressed genes.

metallothionein (Mt)2; suppressor of cytokine signaling 3; JunB proto-oncogene, AP-1 transcription factor subunit (Junb); tribbles pseudokinase 3; CCAAT/enhancer binding protein $\beta$ (Cebpb); nocturnin; methylenetetrahydrofolate dehydrogenase (NADP+ dependent)2, methenyltetrahydrofolate cyclohydrolase; Cxcl1; basic helix-loop-helix family member e40; Mt1; intercellular adhesion molecule 1; NLR family CARD domain-containing 5; and immunity-related GTPase family M member 2 (Fig. 4A). However, only 1 downregulated DEG, olfactomedin-like 1 (Olfml1), was identified in all three tissue types (Fig. 4B).

iRegulon analysis revealed that $6 \mathrm{TFs}$ were predicted to regulate DEGs that were common among all three tissue types, including Cebpb (NES=8.957), Stat5a $(\mathrm{NES}=6.51)$, metal regulatory transcription factor 1 (NES=5.431), REL proto-oncogene, nuclear factor- $\kappa \mathrm{B}$ subunit $(\mathrm{NES}=5.177)$, ETS variant 6 (NES=5.154) and Sp1 transcription factor (NES=5.119). Cebpb and Stat5a were indicated to modulate 10 DEGs, including Len2 and Cxcl1, and Olfml1, Cxcl1 and Junb, respectively (Fig. 4C).

\section{Discussion}

MODS is a major cause of morbidity and mortality among patients with acute physiological damages $(3,4)$. In the present study, based on the analysis of the gene expression profile of mice with MODS compared with healthy controls, 943 , 267 and 227 DEGs were identified in lung, liver and kidney samples, respectively. Pathway enrichment analysis of DEGs revealed that several DEGs that were common among lung and liver tissues were implicated in 'cytokine-cytokine receptor interaction' and the 'Jak-STAT signaling pathway'.

Cxcl1 and Cxcl10, DEGs that were identified in lung and liver tissue, are implicated in the "cytokine-cytokine receptor interaction' pathway. During the immune response in MODS, cytokine secretion is reported to cause universal endothelial injury in organs, a pathological process that results in MODS (5). Cxcl1 and Cxcl10 encode CXC subfamily inflammatory chemokines $(29,30)$. Upon binding to CXC receptor (CXCR) 3, Cxcl10 induced the recruitment of natural killer effectors, including $\mathrm{T}$ cells and dendritic cells (31). During the early stages of multiple organ failure (MOF), Cxcl10 expression was significantly increased compared with the non-MOF control (32), which is consistent with the results of the present study. In the PPI network of DEGs in the lungs and liver, Cxcl10 exhibited one of the highest degrees and interacted with Cxcl1. The receptor for Cxcl1, CXCR2, is hypothesized to act as a potential therapeutic target in sepsis, and the outcomes of the disease were improved in CXCR2-knockout mice with severe sepsis (33). There is evidence indicating that a CXCR2 chemokine receptor antagonist protected mice from acute pancreatitis and lung injury (34). The above studies indicate that CXCR2 and its ligand Cxcl1 may serve a role in the immune response in sepsis and organ failure. Taken together, Cxcl1 and Cxcl10 may exert functions in the progression of MODS.

Stat1, implicated in the Jak-STAT signaling pathway, demonstrated the highest degree in the PPI network for lung and liver tissue. Stat 1 encodes a protein member of the STAT family, which mediates cellular responses to cytokines and growth factors (35). Stat1 is activated by various cytokines and is associated with proinflammatory signaling and the development of inflammatory tissue damage (36). In severe acute pancreatitis with organ failure, Stat1 was reported to be activated in monocytes (37). Furthermore, the Jak-STAT signaling pathway has been reported to be implicated in heart failure through the activity of the Stat 3 protein (38). In the PPI network of DEGs in the lungs and liver, Stat1 interacted with Cxcl10. Therefore, Stat1 may serve a role in the progression of MODS via regulation of the Jak-STAT signaling pathway or interaction with Cxcl10.

Two signaling pathways, MAPK and p53 pathways, were common to liver and kidney samples. The results of the present study demonstrated that the DEG Gadd45A was enriched in both pathways. Gadd45A is a p53-regulated stress response protein and its transcription is stimulated by 
Table IV. Top 20 DEG nodes from mice with MODS demonstrating the highest degrees in PPI networks.

A, Top 20 DEG nodes with the highest degrees in the lung PPI network

\begin{tabular}{lc}
\hline Node & Degree \\
\hline Stat1 & 99 \\
Tnf & 71 \\
IL-6 & 64 \\
Ccr7 & 57 \\
Cxcl10 & 54 \\
Irf1 & 50 \\
Mmp9 & 47 \\
Irf7 & 46 \\
Cxcr2 & 46 \\
Gbp2 & 45 \\
Ccr5 & 44 \\
Ifit1 & 43 \\
Ccr1 & 43 \\
Ifit3 & 42 \\
Ccr2 & 42 \\
Oas12 & 41 \\
Cxcr6 & 41 \\
Icam1 & 40 \\
Oas11 & 41 \\
Tyrobp & 40 \\
\hline & \\
\hline
\end{tabular}

B, Top 20 DEG nodes with the highest degrees in the liver PPI network

\begin{tabular}{lc}
\hline Node & Degree \\
\hline Jun & 16 \\
Stat3 & 16 \\
Stat1 & 16 \\
Icam1 & 12 \\
Gbp2 & 11 \\
Cxc110 & 11 \\
Irf9 & 10 \\
Egr1 & 9 \\
Cyp7a1 & 9 \\
Sirt1 & 9 \\
Ifit1 & 8 \\
Usp18 & 8 \\
Ifit3 & 8 \\
Hsd3b5 & 8 \\
Cyp2b10 & 8 \\
Cyp3a13 & 8 \\
Rsad2 & 8 \\
Irgm2 & 7 \\
Saa1 & 7 \\
Saa2 & 7 \\
&
\end{tabular}

Table IV. Continued.

C, Top 20 DEG nodes with the highest degrees in the kidney PPI network

\begin{tabular}{|c|c|}
\hline Node & Degree \\
\hline Jun & 28 \\
\hline Fos & 27 \\
\hline IL-6 & 23 \\
\hline Myc & 20 \\
\hline Egr1 & 20 \\
\hline Stat3 & 17 \\
\hline Atf3 & 14 \\
\hline Sirt1 & 14 \\
\hline Ptgs2 & 14 \\
\hline Adipoq & 13 \\
\hline Cdkn1a & 12 \\
\hline Zfp36 & 11 \\
\hline Btg2 & 10 \\
\hline Hmox1 & 10 \\
\hline Cebpb & 10 \\
\hline Junb & 9 \\
\hline Egr2 & 9 \\
\hline Icam1 & 8 \\
\hline Ddit3 & 8 \\
\hline Retn & 7 \\
\hline Klf4 & 7 \\
\hline
\end{tabular}

DEGs, differentially expressed genes; MODS, multiple organ dysfunction syndrome; PPI, protein-protein interaction.

stress-associated conditions inhibiting growth and by exposure to DNA-damaging agents $(39,40)$. Gadd45A has been demonstrated to be highly expressed in a mouse model of lung injury (41). In addition, upregulated Gadd45A has been detected in autoimmune diseases and contributed to autoimmunity by promoting DNA demethylation in T cells (39). Gadd45A is able to activate MEK kinase 4, which results in the phosphorylation and activation of the MAPKs p38 and c-Jun $\mathrm{N}$-terminal kinase during an inflammatory response $(42,43)$. It has been reported that MAPKs are upregulated in the toll-like receptor 4-mediated progression of multi-organ failure (44). Furthermore, p53 has been demonstrated to serve a role in inflammation (45). Therefore, Gadd45Amay function in the progression of MODS through regulation of the MAPK and p53 signaling pathways.

In lung, liver and kidney samples, there were 18 common DEGs, including 17 upregulated genes and 1 downregulated gene. Among the 17 upregulated genes, Cebpb was identified as a TF and regulated multiple DEGs that were common among the three tissue types, including Cxcl1. Cebpb encodes a transcription factor that contains a basic leucine zipper domain and functions in the regulation of genes associated with immune and inflammatory responses (46). It has been 
A

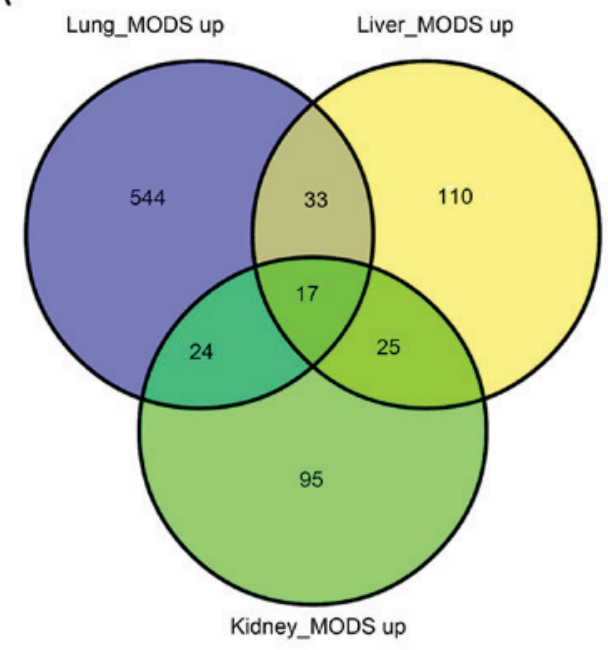

B

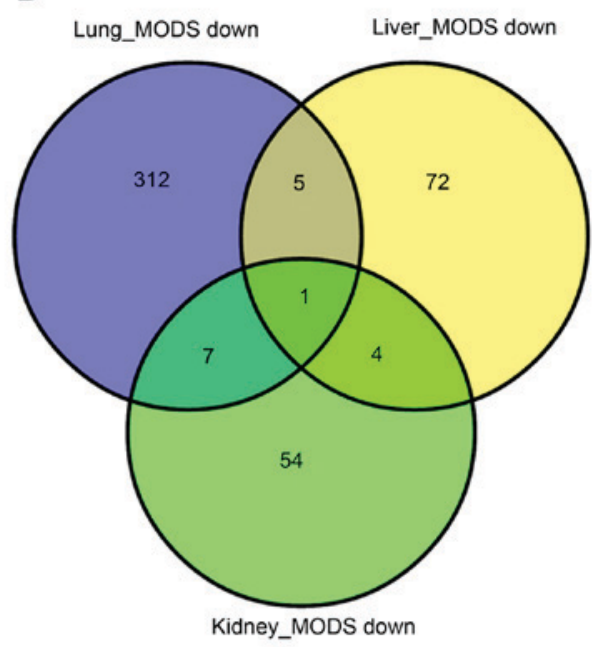

C

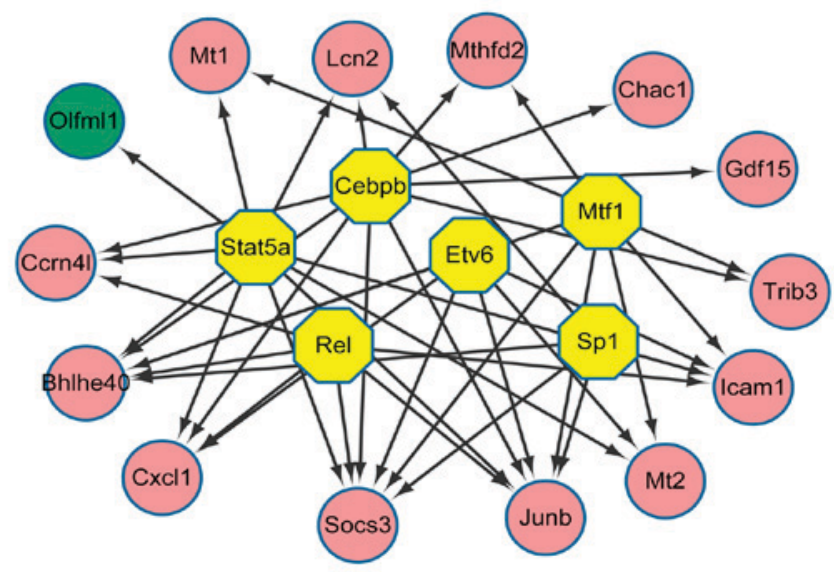

Figure 4. Diagrams representing DEGs in lung, liver and kidney samples, and a regulatory network of common genes and TFs. Venn diagrams presenting (A) upregulated and (B) downregulated DEGs in lung, liver and kidney samples from mice with MODS. (C) Regulatory network of DEGs and TFs that were common among the three types of organ samples. MODS, multiple organ dysfunction syndrome; DEGs, differentially expressed genes; TFs, transcription factors; MODS, multiple organ dysfunction syndrome; up, upregulated genes; down, downregulated genes.

demonstrated that Cebpb in the lung epithelium contributed to lipopolysaccharide-induced Cxcl1 expression. There is no direct evidence to confirm a role for Cebpb in MODS. Therefore, the present study hypothesized that Cebpb may serve a role in the progression of MODS as a TF regulating the expression of Cxcl1 and other genes.

Additionally, in the three organs analyzed in the present study, the only common downregulated gene, Olfmll, is a glycoprotein containing the olfectamine domain. In neural tissues, olfactomedin was associated with the growth and differentiation of chemosensory cilia (47). To the best of our knowledge, an association between Olfmll and MODS has not been previously reported. In the present study, Olfml1 was regulated by Stat5a, a member of the STAT family transcription factors. Stat5a functions in immunity by regulating the development and maintenance of $\mathrm{T}$ regulatory cells (48-50). Therefore, Olfml1 may be implicated in the progression of MODS through regulation by Stat5a.

There were several limitations of the present study. The predicted results should be confirmed by laboratory data. Furthermore, in the present study, a limited number of samples were used, which should be increased to improve the reliability of the conclusions drawn. In future studies, a higher number of organ samples from mice with MODS will be included to validate the expression levels of potentially implicated genes and to elucidate their functions.

In conclusion, 943, 267 and 227 DEGs were identified in lung, liver and kidney samples from mice with MODS, respectively, compared with the normal healthy controls. Certain DEGs were implicated in pathways that were common to both lung and liver samples, including "cytokine-cytokine receptor interaction' (such as Cxcl1 and Cxcl10) and the 'Jak-STAT signaling pathway' (such as Stat1). In addition, several DEGs, including Gadd45A, were common to two signaling pathways in liver and kidney samples. Furthermore, 18 DEGs were common to all three types of tissues analyzed in the present study, including Cebpb and Olfml1. Cebpb regulated multiple DEGs, such as Cxcl1, and Stat5a regulated the expression of Olfml1. The genes and pathways identified in the present study may be implicated in the progression of MODS. Therefore, the results of the present study may provide potential therapy targets for the treatments of MODS. 


\section{Acknowledgements}

The present study was supported by the Scientific and Technological Project in the Social Development Area of Science and Technology Department of Shaanxi Province (grant no. 2012K16-12-02).

\section{References}

1. Volman TJ, Hendriks T and Goris RJ: Zymosan-induced generalized inflammation: Experimental studies into mechanisms leading to multiple organ dysfunction syndrome. Shock 23: 291-297, 2005

2. Henao FJ, Daes JE and Dennis RJ: Risk factors for multiorgan failure: A case-control study. J Trauma 31: 74-80, 1991.

3. Deitch EA: Multiple organ failure. Pathophysiology and potential future therapy. Ann Surg 216: 117-134, 1992.

4. Marshall JC: Inflammation, coagulopathy, and the pathogenesis of multiple organ dysfunction syndrome. Crit Care Med 29 (7 Suppl): S99-S106, 2001.

5. Wang $\mathrm{H}$ and Ma S: The cytokine storm and factors determining the sequence and severity of organ dysfunction in multiple organ dysfunction syndrome. Am J Emerg Med 26: 711-715, 2008.

6. Gustot T: Multiple organ failure in sepsis: Prognosis and role of systemic inflammatory response. Curr Opin Crit Care 17: 153-159, 2011.

7. Henninger DD, Panés J, Eppihimer M, Russell J, Gerritsen M Anderson DC and Granger DN: Cytokine-induced VCAM-1 and ICAM-1 expression in different organs of the mouse. J Immunol 158: 1825-1832, 1997.

8. Bratt $\mathbf{J}$ and Palmblad J: Cytokine-induced neutrophil-mediated injury of human endothelial cells. J Immunol 159: 912-918, 1997.

9. Crouser ED: Mitochondrial dysfunction in septic shock and multiple organ dysfunction syndrome. Mitochondrion 4: 729-741, 2004.

10. Suzuki T, Takahashi T, Yamasaki A, Fujiwara T, Hirakawa M and Akagi R: Tissue-specific gene expression of heme oxygenase-1 (HO-1) and non-specific delta-aminolevulinate synthase (ALAS-N) in a rat model of septic multiple organ dysfunction syndrome. Biochem Pharmacol 60: 275-283, 2000.

11. Rockey DC, Bell PD and Hill JA: Fibrosis-a common pathway to organ injury and failure. N Engl J Med 372: 1138-1149, 2015.

12. Bunney WE, Bunney BG, Vawter MP, Tomita H, Li J, Evans SJ, Choudary PV, Myers RM, Jones EG, Watson SJ and Akil H: Microarray technology: A review of new strategies to discover candidate vulnerability genes in psychiatric disorders. Am J Psychiatry 160: 657-666, 2003.

13. Nylund L, Satokari R, Nikkilä J, Rajilić-Stojanović M, Kalliomäki M, Isolauri E, Salminen $S$ and De Vos WM: Microarray analysis reveals marked intestinal microbiota aberrancy in infants having eczema compared to healthy children in at-risk for atopic disease. BMC Microbiol 13: 12, 2013.

14. Maslove DM and Wong HR: Gene expression profiling in sepsis: Timing, tissue, and translational considerations. Trends Mol Med 20: 204-213, 2014.

15. Gutschner T, Hämmerle M, Eissmann M, Hsu J, Kim Y, Hung G, Revenko A, Arun G, Stentrup M, Gross M, et al: The noncoding RNA MALAT1 is a critical regulator of the metastasis phenotype of lung cancer cells. Cancer Res 73: 1180-1189, 2013.

16. Di Narzo AF, Tejpar S, Rossi S, Yan P, Popovici V, Wirapati P, Budinska E, Xie T, Estrella H, Pavlicek A, et al: Test of four colon cancer risk-scores in formalin fixed paraffin embedded microarray gene expression data. J Natl Cancer Inst 106: pii: dju247, 2014.

17. Gharib SA, Mar D, Bomsztyk K, Denisenko O, Dhanireddy S, Liles WC and Altemeier WA: System-wide mapping of activated circuitry in experimental systemic inflammatory response syndrome. Shock 45: 148-156, 2016.

18. Gautier L, Cope L, Bolstad BM and Irizarry RA: affy-analysis of Affymetrix GeneChip data at the probe level. Bioinformatics 20 : 307-315, 2004

19. Carlson M, Falcon S, Pages H and Li N: org. Mm. eg. db: Genome wide annotation for Mouse. org. Mm. eg. db: Genome wide annotation for Mouse. R package version, 2012.

20. Carlson M: Mouse4302.db: Affymetrix Mouse genome 4302.0 array annotation data (chip mouse4302). $\mathrm{R}$ package version 3.1.3, 2016.
21. Smyth GK: Limma: linear models for microarray data. In: Bioinformatics and Computational Biology Solutions Using $\mathrm{R}$ and Bioconductor. Gentleman R, Carey V, Huber W, Irizarry R and Dudoit S (eds). Springer, New York, NY, pp397-420, 2005.

22. Glueck DH, Mandel J, Karimpour-Fard A, Hunter L and Muller KE: Exact calculations of average power for the Benjamini-Hochberg procedure. Int J Biostat 4: Article 11, 2008.

23. Warnes GR, Bolker B, Bonebakker L, Gentleman R, Huber W, Liaw A, Lumley T, Maechler M, Magnusson A, Moeller S, et al: gplots: Various R programming tools for plotting data. R package version 2, 2009.

24. Kolde R: Pretty heatmaps, 2015. https://cran.r-project.org/web/ packages/pheatmap/pheatmap.pdf. Accessed December 11, 2015

25. Huang DW, Sherman BT, Tan Q, Collins JR, Alvord WG, Roayaei J, Stephens R, Baseler MW, Lane HC and Lempicki RA: The DAVID gene functional classification tool: A novel biological module-centric algorithm to functionally analyze large gene lists. Genome Biol 8: R183, 2007.

26. Szklarczyk D, Franceschini A, Wyder S, Forslund K, Heller D, Huerta-Cepas J, Simonovic M, Roth A, Santos A, Tsafou KP, et al: STRING v10: Protein-protein interaction networks, integrated over the tree of life. Nucleic Acids Res 43(Database Issue): D447-D452, 2015.

27. Kohl M, Wiese S and Warscheid B: Cytoscape: Software for visualization and analysis of biological networks. Methods Mol Biol 696: 291-303, 2011.

28. Janky R, Verfaillie A, Imrichová H, Van de Sande B, Standaert L, Christiaens V, Hulselmans G, Herten K, Naval Sanchez M, Potier D, et al: iRegulon: From a gene list to a gene regulatory network using large motif and track collections. PLoS Comput Biol 10: e1003731, 2014

29. Booth V, Keizer DW, Kamphuis MB, Clark-Lewis I and Sykes BD: The CXCR3 binding chemokine IP-10/CXCL10: Structure and receptor interactions. Biochemistry 41: 10418-10425, 2002.

30. Haskill S, Peace A, Morris J, Sporn SA, Anisowicz A, Lee SW, Smith T, Martin G, Ralph P and Sager R: Identification of three related human GRO genes encoding cytokine functions. Proc Natl Acad Sci USA 87: 7732-7736, 1990.

31. Panzer U, Steinmetz OM, Paust HJ, Meyer-Schwesinger C, Peters A, Turner JE, Zahner G, Heymann F, Kurts C, Hopfer $\mathrm{H}$, et al: Chemokine receptor CXCR3 mediates $\mathrm{T}$ cell recruitment and tissue injury in nephrotoxic nephritis in mice. J Am Soc Nephrol 18: 2071-2084, 2007.

32. Jastrow KM III, Gonzalez EA, McGuire MF, Suliburk JW, Kozar RA, Iyengar S, Motschall DA, McKinley BA, Moore FA and Mercer DW: Early cytokine production risk stratifies trauma patients for multiple organ failure. J Am Coll Surg 209: 320-331, 2009.

33. Robertson CM and Coopersmith CM: The systemic inflammatory response syndrome. Microbes Infect 8: 1382-1389, 2006.

34. Bhatia M and Hegde A: Treatment with antileukinate, a CXCR2 chemokine receptor antagonist, protects mice against acute pancreatitis and associated lung injury. Regul Pept 138: 40-48, 2007.

35. Quelle FW, Thierfelder W, Witthuhn BA, Tang B, Cohen S and Ihle JN: Phosphorylation and activation of the DNA binding activity of purified Stat 1 by the Janus protein-tyrosine kinases and the epidermal growth factor receptor. J Biol Chem 270: 20775-20780, 1995.

36. Durbin JE, Hackenmiller R, Simon MC and Levy DE: Targeted disruption of the mouse Stat 1 gene results in compromised innate immunity to viral disease. Cell 84: 443-450, 1996.

37. Oiva J, Mustonen $H$, Kylänpää $M L$, Kyhälä L, Alanärä $T$, Aittomäki S, Siitonen S, Kemppainen E, Puolakkainen P and Repo H: Patients with acute pancreatitis complicated by organ failure show highly aberrant monocyte signaling profiles assessed by phospho-specific flow cytometry. Crit Care Med 38: 1702-1708, 2010.

38. Boengler K, Hilfiker-Kleiner D, Drexler H, Heusch G and Schulz R: The myocardial JAK/STAT pathway: From protection to failure. Pharmacol Ther 120: 172-185, 2008.

39. Li Y, Zhao M, Yin H, Gao F, Wu X, Luo Y, Zhao S, Zhang X, $\mathrm{Su} \mathrm{Y}, \mathrm{Hu} \mathrm{N}$, et al: Overexpression of the growth arrest and DNA damage-induced 45alpha gene contributes to autoimmunity by promoting DNA demethylation in lupus T cells. Arthritis Rheum 62: 1438-1447, 2010.

40. Zhan Q: Gadd45a, a p53-and BRCA1-regulated stress protein, in cellular response to DNA damage. Mutat Res 569: 133-143, 2005.

41. Altemeier WA, Matute-Bello G, Gharib SA, Glenny RW, Martin TR and Liles WC: Modulation of lipopolysaccharide-induced gene transcription and promotion of lung injury by mechanical ventilation. J Immunol 175: 3369-3376, 2005. 
42. Uhlig U, Haitsma JJ, Goldmann T, Poelma DL, Lachmann B and Uhlig S: Ventilation-induced activation of the mitogen-activated protein kinase pathway. Eur Respir J 20: 946-956, 2002.

43. Li LF, Yu L and Quinn DA: Ventilation-induced neutrophil infiltration depends on c-Jun N-terminal kinase. Am J Respir Crit Care Med 169: 518-524, 2004.

44. McGhan LJ and Jaroszewski DE: The role of toll-like receptor-4 in the development of multi-organ failure following traumatic haemorrhagic shock and resuscitation. Injury 43: 129-136, 2012.

45. Cooks T, Harris CC and Oren M: Caught in the cross fire: p53 in inflammation. Carcinogenesis 35: 1680-1690, 2014.

46. Wang W, Bergh A and Damber JE: Increased expression of CCAAT/enhancer-binding protein beta in proliferative inflammatory atrophy of the prostate: Relation with the expression of COX-2, the androgen receptor, and presence of focal chronic inflammation. Prostate 67: 1238-1246, 2007.

47. Bal RS and Anholt RR: Formation of the extracellular mucous matrix of olfactory neuroepithelium: Identification of partially glycosylated and nonglycosylated precursors of olfactomedin. Biochemistry 32: 1047-1053, 1993.
48. Takatori H, Nakajima H, Kagami S, Hirose K, Suto A, Suzuki K, Kubo M, Yoshimura A, Saito Y and Iwamoto I: Stat5a inhibits IL-12-induced Th1 cell differentiation through the induction of suppressor of cytokine signaling 3 expression. J Immunol 174: 4105-4112, 2005.

49. Takatori H, Nakajima H, Hirose K, Kagami S, Tamachi T, Suto A, Suzuki K, Saito Y and Iwamoto I: Indispensable role of Stat5a in Stat6-independent Th2 cell differentiation and allergic airway inflammation. J Immunol 174: 3734-3740, 2005.

50. Wei L, Laurence A and O'Shea JJ: New insights into the roles of Stat $5 \mathrm{a} / \mathrm{b}$ and Stat 3 in T cell development and differentiation. Semin Cell Dev Biol 19: 394-400, 2008.

(7) (9) This work is licensed under a Creative Commons Attribution-NonCommercial-NoDerivatives 4.0 International (CC BY-NC-ND 4.0) License. 